\title{
Long-term survival after corrective surgeries in two patients with severe deformities due to Roberts syndrome: A Case report and review of the literature
}

\author{
JING ZHOU* ${ }^{*}$ XIAONAN YANG* ${ }^{*}$ XIAOLEI JIN, ZHENHUA JIA, \\ HAIBIN LU and ZUOLIANG QI
}

Plastic Surgery Hospital, Chinese Academy of Medical Sciences, Peking Union Medical College, Beijing 100144, P.R. China

Received June 27, 2017; Accepted October 25, 2017

DOI: $10.3892 /$ etm.2017.5592

\begin{abstract}
Roberts syndrome (RBS; OMIM 268300) is a rare autosomal recessive disease characterized by retardation before and after birth, cranial and maxillofacial deformities, limb anomalies and intellectual disability. Mutations in the establishment of cohesion 1 homologue 2 (ESCO2) gene on chromosome 8 p21.1 have been found to be causative for RBS. We describe two patients with RBS with physical deformities and 11. One is an 8-year-old Yemeni male, and the other is his 13-year-old sister. These patients were diagnosed with RBS and underwent surgeries during their first to third years of life. Here, we present the cases for the two patients, focusing specifically on their surgical management and outcomes. Additionally, by reviewing the literature on RBS, we also summarize the proper surgical interventions for this rare disease. This paper describes the long-term follow-up of two patients with severe deformities who benefitted from corrective surgeries. The findings of this study indicate that patients who survive infancy and reach adulthood, even patients who present with severe disease symptoms, can benefit from corrective surgeries and lead better lives.
\end{abstract}

\section{Introduction}

Roberts syndrome (RBS) was first described by Roberts in 1919 (1) and is an extremely rare condition that has been reported in only approximately 150 individuals of diverse racial and ethnic backgrounds. The frequency of RBS carriage is unknown (2);

Correspondence to: Dr Zuoliang Qi, Plastic Surgery Hospital, Chinese Academy of Medical Sciences, Peking Union Medical College, 33 Badachu Road, Shijingshan, Beijing 100144, P.R. China

E-mail: public-qi@163.com

*Contributed equally

Abbreviations: RBS, Roberts syndrome; SC, phocomelia syndrome

Key words: Roberts syndrome, rare disease, surgical treatment, maxillofacial deformities, limb anomalies however, parental consanguinity is common among individuals affected by this genetic disorder. It has been verified that RBS and SC phocomelia syndrome are the same disease but present with different severities and that the deformity characteristic of both diseases is more severe in the former condition than in the latter (3). The literature on RBS indicates that the disease presents with a variety of signs, symptoms and abnormalities, including growth retardation, limb defects, craniofacial anomalies and multi-organ dysfunction. In most cases, affected patients succumb to respiratory and cardiac failure. However, some patients with mild symptoms may survive.

The causative gene for RBS is establishment of cohesion 1 homologue 2 (ESCO2), which encodes a protein comprising 601 amino acids belonging to the Ecol family of acetyltransferases. These enzymes participate in the establishment of sister chromatid cohesion during $\mathrm{S}$ phase and in post-replicative sister chromatid cohesion induced by double-strand breaks (4-6). The process of cohesion may be regulated either directly or indirectly by the acetylation activity of these proteins (7). Loss of ESCO2 acetyltransferase activity may be the molecular mechanism underlying RBS development (8).

Here, we report the medical histories of two children of a consanguineous couple with RBS (Fig. 1) and provide a review of the current clinical and genetic features of the condition. Additionally, we discuss appropriate therapeutic surgeries with which the disease can be managed.

\section{Patients and methods}

Patients. This study was reviewed and approved by the Peking Union Medical College Hospital Ethics Review Board (Beijing, China). Informed consents were obtained from the legal guardian of the two patients. Both participants' legal guardians provided their written informed consents to participate on behalf of the children. We obtained written informed consent for the publication of any associated data and accompanying images and maximally hide other unidentifiable features, thus protecting the privacy for each patient.

Literature review of $R B S$. The authors searched the published literature in PubMed using the following search keys: ('Roberts syndrome' OR 'SC Phocomelia syndrome') 
respectively, without language restriction, with published data up to October 26, 2016. Together, we found 186 papers. Among them, titles of 124 papers were related to the subject. After reading the abstract, we found 65 papers reporting 125 cases. By reviewing these articles, we searched referenced cases reported earlier either through the library of Peking Union Medical College or by inter-library loan. Together, we found 137 cases and by reading these cases, we throughly understood the typical symptoms of RBS patients and recognized the potential symptoms of adult slightly affected cases. Further, we extracted information of $17 \mathrm{RBS}$ patients who received surgical treatment and analyzed the operating modus, juncture and outcomes of their surgeries. By doing this, we came to a suggestion over the suitable operation time and their potential outcomes.

\section{Results}

Case 1. The male patient was full-term and delivered normally and was born with physical deformities involving several areas of his body, especially his head and limbs. The child physical and mental development was significantly delayed. Between 1 and 2 years of age, the patient underwent cleft lip and palate surgery and frontal fenestration surgery under general anesthesia. Post-surgery, the patient's condition improved; however, he still presented with oral dyskinesia, secondary cranial deformities and other anomalies.

Upon admission, the 8-year-old male patient was $69 \mathrm{~cm}$ tall, weighed $13 \mathrm{~kg}$ and displayed global developmental delays. He could stand but could barely walk. His eyes were unfocused, and he had unclear speech. Moreover, he was not very cooperative during the physical examination. The patient presented with sparse dark head hair; thus, a coronary osseous contracture zone was visible in the center of the parietal region. The protruding tissue in the midline area of the forehead was soft and beat regularly. The anteroposterior diameter of the head was longer than the corresponding biparietal diameter. The patient also had apparent bilateral exophthalmos and incomplete closure of the palpebral fissures. As the patient's head was too small, a regular exopthalmometer could barely measure eye protrusion. The central face was smooth, as the nasal columella and alae did not appear to be clearly defined. An incision scar was visible on the skin adjacent to the protruding upper lip. The side of the tongue adhered tightly to the alveolar bone, resulting in movement limitation, lip closure failure and irregular tooth alignment. The patient's upper arms displayed seal-limb deformities. Moreover, each hand had four fingers and exhibited syndactylism. The patient had short lower limbs that buckled, and his knee joints did not display any functional movement. No obvious abnormalities of the external genitalia were observed (Fig. 2). The patient has no self-care ability and is completely dependent on others.

A skeletal survey showed several abnormalities. For example, the skull film showed evidence of micrognathia. There was marked thinning with mild broadening of the skull vault (Fig. 3). The lumbar spine showed molded vertebral bodies. Multiple appendicular skeletal anomalies were also noted, including bizarrely shaped bilateral humeri and absent radii and ulnae bilaterally. Additionally, only three metacarpal bones were present bilaterally, and only four fingers were present on each side. No tibial or fibular structures were observed in the lower limbs, and the long bones were fused angularly at the knee joints. A CT scan of the brain demonstrated the following abnormalities: craniosynostosis mainly affecting the coronal and the lambdoid sutures, as well as small orbital cavities and a stenotic skull base (Fig. 3). The brain parenchyma showed at least two foci of dystrophic calcification in the left frontal and parietal lobes. These may had been caused by an intrauterine infection. A CT of the chest and heart indicated that the left 12 th rib was absent. Both lung fields were clear. The cardiac shadow was within normal limits with respect to its size and configuration. Echocardiography demonstrated a congenital heart defect (a patent ductus arteriosus). Cytogenetic analysis revealed a normal karyotype with premature centromere separation (PCS). PCS, also known as heterochromatin repulsion (HR), is an abnormality of sister chromatid apposition around the centromere that is particularly noticeable in chromosomes with large blocks of heterochromatin (chromosomes 1, 16 and 22) (Fig. 1).

The patient was admitted to the hospital for treatment of the bilateral exophthalmos and improvement of his abnormal palpebral fissures. After admission, we held a multi-disciplinary meeting involving doctors from several hospitals and decided to perform a cranial cavity enlargement, skull rearrangement and frontal orbital transferring surgery. However, not long after admission, the patient presented with signs and symptoms of a severe upper respiratory infection. Due to his poor physical condition, the patient was discharged from our hospital and returned to Yemen for his recovery.

Case 2. The 13-year-old female patient was full-term and delivered normally and presented with deformities in the craniofacial region and the limbs. She showed milder growth and intellectual disability than her younger brother. Between 1 and 2 years of age, the patient underwent cleft lip and palate repair under general anesthesia. She also underwent a talipes equinovarus operation at the age of 3 .

On physical examination, the patient had short stature. Her height was $122 \mathrm{~cm}(<3 \mathrm{rd}$ percentile for a 13-year-old female child), and her weight was $28.5 \mathrm{~kg}$. Her speech was slurred but was understandable. The patient presented with an apparent scar from the cleft lip and palate repair, as well as hypoplastic alae nasi (Fig. 4). The patient had a large mandible accompanied by a short maxillary bone and displayed malocclusion whose features were consistent with class III disease, or mesiocclusion. She had full range of motion of her shoulders and wrists, but displayed symmetric fusion of her elbows, which did not appear to be capable of functional movement. The patient presented with symmetrical dysplasia of her first metacarpal bone and first proximal and distal phalanx, anomalies accompanied by clinodactyly of the second and fifth fingers (Fig. 5). Both lower limbs were short. The left leg was shorter than the right. The right knee was fused and could not flex. The patient had a history of a metatarsus varus deformity of the right foot that had been successfully treated with casts; however, she remained flat-footed. Moreover, her gait had improved after the operation but was not entirely normal. The patient's cardiac, renal and pulmonary function was normal. Cytogenetic analysis revealed a normal karyotype with PCS involving chromosomes 1, 16 and 22. (Fig. 1). The patient had the intellectual ability of a 


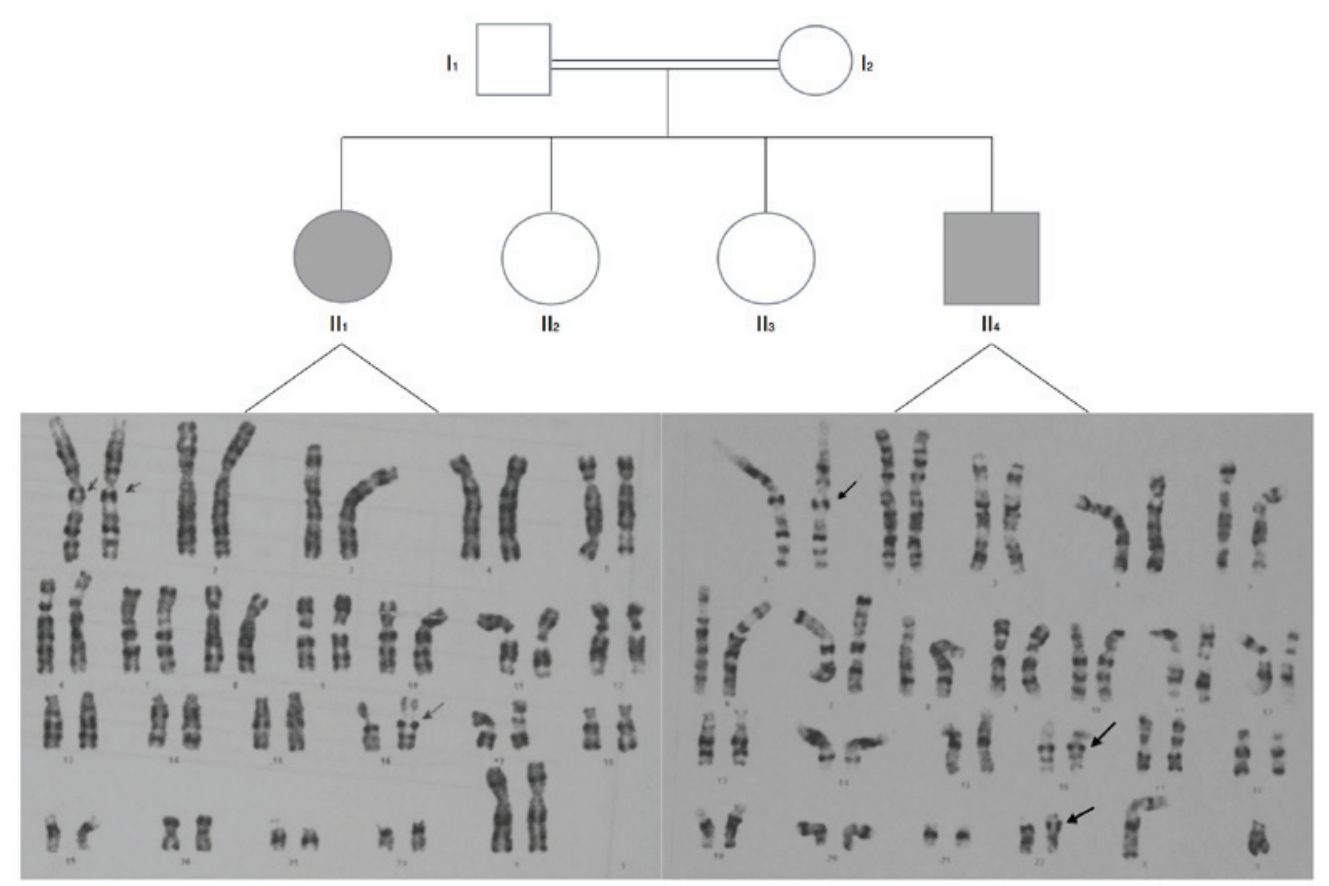

Figure 1. Family tree and G-banded chromosomes results of II1 and II4, showing abnormal morphological features indicative of premature centromere separation. Arrows indicate premature centromere sepaprations in chromosomes 1 and 16 in the female patient, and chromosomes 1, 16 and 22 in the male patient.

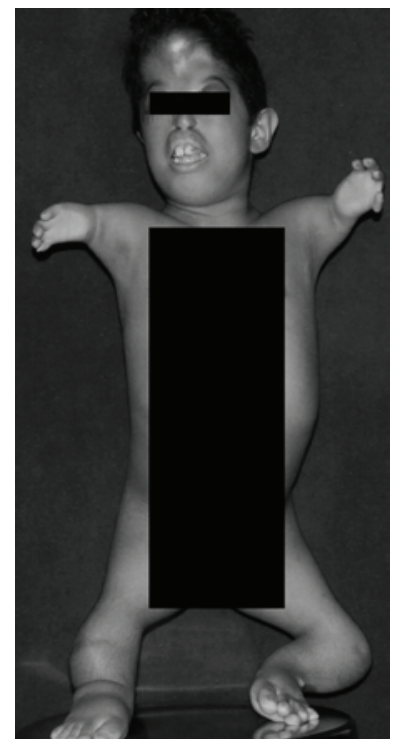

Figure 2. General view of the male patient. Protruding tissue in the midline area of the forehead. Visible scar adjacent to upper lip. Lips closure failure and irregular tooth alignment. Seal-limb deformities with syndactylism. Buckled lower limbs with ankylosis of knees.

7-year-old child. She could complete daily tasks and take care of herself. Specifically, she could dress and feed herself. Since she was the first child in her family to present with any birth deformities, neither her parents nor the hospital at which she was born had elected to performed an investigation of her abnormalities until her younger brother exhibited more severe signs and symptoms of the same disease.

The patients' consanguineous (first cousins) Yemeni parents were in good health. The mother had given birth to 4 children ( 3 females and 1 male). The other two sisters did not exhibit any deformities. The parents denied any family history

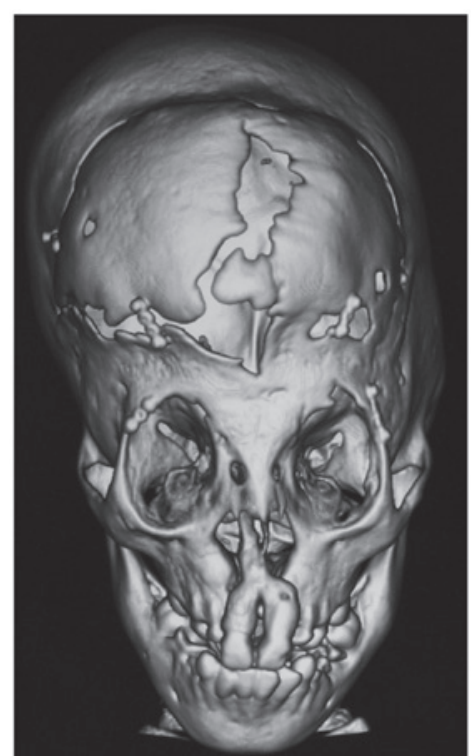

Figure 3. Anteroposterior view of head CT with three-dimensional reconstruction of male patient. Craniosynostosis of coronal and the lambdoid sutures. Small orbital cavities and a stenotic skull base.

of congenital diseases, as well as any history of drug exposure during the abovementioned pregnancies.

Literature review and case discussion. RBS is a rare autosomal recessive disorder, as only approximately 150 cases of the disease have been reported in the literature thus far. The distribution of RBS is global, and the disease exhibits no racial predilection. All reported patients experienced prenatal growth retardation. Other manifestations of the disease, such as postnatal retardation, craniofacial deformities, and limb anomalies, vary with respect to their frequency in affected patients. 


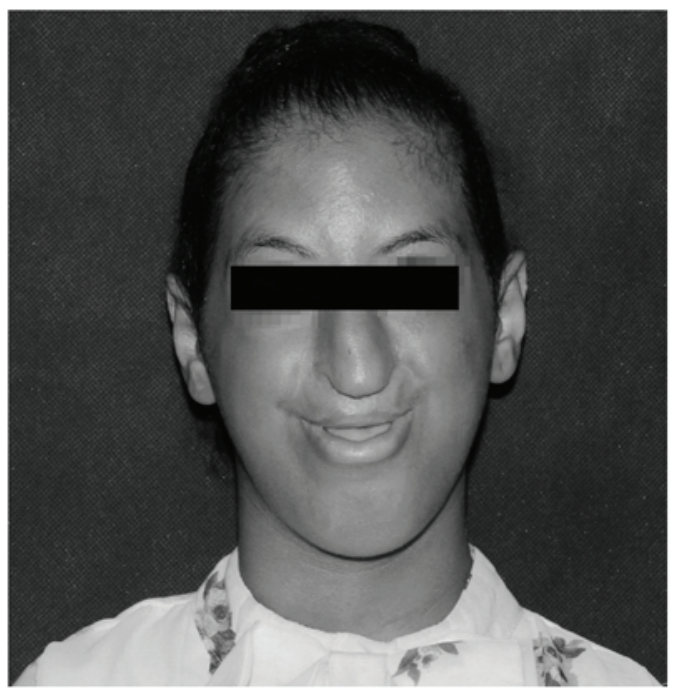

Figure 4. Head general view of the female patient. Hypoplastic alae nasi. Scars near the upper lip.

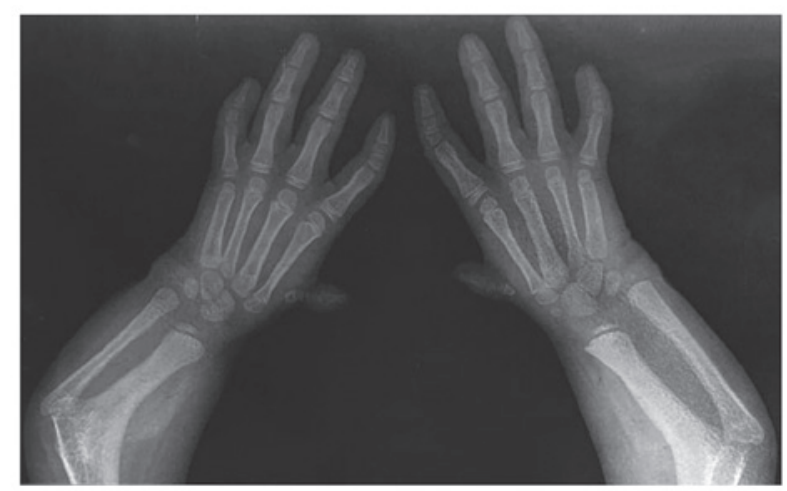

Figure 5. X-ray images of both hands of the female patient. Symmetrical dysplasia of first metacarpal bone and first proximal and distal phalanx. Anomalies accompanied by clinodactyly of the second and fifth fingers.

Clinical features. Prior to the recent discovery of the ESCO2 mutation, which is the causative mutation for RBS, the disease was diagnosed based on patient phenotypic characteristics. According to previous reports, prenatal growth retardation is consistently noted in affected patients, and postnatal growth delay also occurs in some patients. The limb deformities associated with the disease include completely absent arms and legs, as well as arms and legs with rudimentary digits. Mildly reduced limb growth, bone fusions and flexion contractures are also commonly observed (9). In addition, the upper limbs are often more severely affected than the lower limbs. The craniofacial malformations (10) characteristic of the disease include microcephaly, micrognathia, cleft lip and/or palate, encephaloceles, prominent maxilla, exophthalmos, hypertelorism, corneal clouding, a wide nasal bridge, telecanthus, hypoplastic nasal alae, and hemangioma. Internal abnormalities of the heart (11), lungs and kidneys (12); enlarged phalluses and clitorises; and abnormalities of the hematopoietic system (13) have also been noted sporadically.

As RBS exhibits a great degree of phenotypic variability even within families, creating criteria for the definition of RBS was necessary. In 1977, the R:S ratio was introduced as a criterion for the diagnosis of RBS/SC. Additional criteria for the diagnosis of RBS include decreased birth length, neonatal survival, cleft lip, cleft palate, decreased upper limb length, decreased lower limb length, the presence of slivery blond hair and/or facial hemangioma, and hypoplastic nasal alae (14). However, classifying patients into these two categories was not sufficient for assessing the severities of their conditions, which correlated strongly with survival. Therefore, based on their review of 100 cases, Van Den Berg and Francke (15) proposed an RS rating system to intended to quantify the severity of the abovementioned malformations. According to this rating system, there are 6 criteria with which disease severity can be quantified, including growth retardation, phocomelia of the arms, phocomelia of the legs, survival beyond 1 month of age, and palatal and ocular abnormalities. The cutoff scores for severe, moderate, and mild disease are total scores higher than 0.5 , between 0.5 and -0.5 and lower than -0.5 , respectively.

It should be noted that cardiac defects do not influence the $\mathrm{RS}$ rating scale despite the fact that several studies have reported that heart defects are present in RBS/RS (16) and are usually the primary cause of death in affected patients. In addition, the condition of the pulmonary system also often determines survival in pediatric patients (17). Furthermore, the influence of intellectual development should also be taken into consideration when assessing disease severity. Since mental development is not consistent with physical development (18-20), the wellbeing of affected patients depends heavily on their mental health.

Genetic features. Standard cytogenetic preparations stained using G-banding or C-staining techniques serve as indispensable evidence of the diagnosis of RBS/SC. Typically, staining demonstrates chromosomal abnormalities characteristic of PCS or HR in most chromosomes in all cell cycle phases. The cause of the above phenomenon was not identified until 2005, when Vega et al (21) confirmed that RBS results from a mutation of ESCO2 on chromosome 8p21.1. The ESCO2 protein product is essential for bridging sister chromatids during S phase; thus, mutations in ESCO2 disrupt cohesion. To date, all individuals with a cytogenetic diagnosis of RBS also have mutations of ESCO2, including small intragenic deletions/insertions and missense, nonsense, and splice-site mutations of the gene. However, no exonic or whole-gene deletions/duplications of ESCO2 have been detected among patients with RBS. The mutated mis-segregated chromosomes are sensitive to DNA damaging agents and show mitotic arrest or delay, which results in PCS, lagging chromosomes, aneuploidy, micronuclei, decreased cell proliferation and hypersensitivity to DNA-damaging agents. However, although the genetic cause of RBS has been discovered, the disease phenotype is not strictly related to the genotype. This phenomenon may be attributable to the existence of a compensatory cohesion mechanism in some cells that undergo normal mitotic divisions. These cells exhibit different sensitivities (or redundancies that mitigate the effects of mutations) to ESCO2 mutations (22). Thus, it is unrealistic to predict the phenotype of the disease based on the genotype (23). However, certain correlations between facial malformation severity and limb reduction have been noted Moreover, the occurrence of corneal opacities may result from specific mutations. In 
addition, ESCO2 is expressed at relatively high levels in cells constituting structures in the brain, face, limbs, kidneys and gonads that are affected by RBS (24).

Surgical treatment and follow-up of RBS. Although the criteria for the diagnosis of the disease have been systematized, standardized surgical treatment guidelines for the management of this disease have scarcely been documented. The majority of severe disease cases present as spontaneous abortions or as early deaths after delivery, whereas patients with mild disease are more likely to survive infancy and reach adulthood. Patients who survive infancy require proper interventions so that their quality of life may be improved, and they may fulfill their potential, even as they live with the stigma of RBS (25). Since affected patients usually present with fragile health conditions that are occasionally accompanied by cardiac and renal dysfunction, every anesthesia exposure and surgery may be life threatening. Consequently, surgeons must gain a better understanding of this syndrome and should cooperate with multiple clinical departments while providing exemplary operative cares after affected patients have been systematically assessed.

Previous studies have reported on 17 patients with RBS who were treated with surgery (Table I). The earliest surgeries that patients with RBS undergo are surgical cleft lip and palate repairs, which are initially performed when patients are 10 weeks of age (26). Cleft lips and palates have adverse effects on speech development, hearing, appearance, and psychology (27); therefore, it is necessary to correct these deformities. Generally, cleft lip is often repaired 10 weeks after birth, while cleft palate is usually repaired between 6 and 12 months after birth. Some surgeons believe that the optimal age range for surgery for cleft lip is 3 to 5 months, while the optimal age range for surgery for cleft palate is 18 to 24 months (28). In addition, patients inevitably undergo different combinations of initial surgeries and repeat surgeries as they grow up (29). By repairing cleft lips and palates, surgeons can reconstruct patients' oral function. Postoperative speech therapy is highly recommended for affected patients, especially patients who undergo later surgery (20). Other craniofacial surgeries that treat craniosynostosis and micrognathia are also recommended for affected patients (30). However, these surgeries are rarely performed due to the poor general health conditions of the patients in question. Moreover, the surgeries are associated with long operative times and increased hospital costs, as well as relatively high risks of complications. However, the patient in case 1 underwent craniofacial surgery, and his long-term follow-up results indicate that the above surgeries can improve quality of life without compromising life expectancy. Therefore, as a result of advancements in medical techniques and the development of sophisticated medical equipment, patients with severe deformities are likely to survive longer than before. Operations that were once regarded as risky should be given more consideration, as they may improve the quality of life of patients with RBS.

RBS is characterized by a high rate of limb abnormalities, and affected patients frequently undergo orthopedic surgeries. Hand surgeries facilitate early development of the prehensile grasp and contribute to improvements in motor function in the future (14). Surgical correction of the lower limbs is also performed to facilitate normal motor development $(31,32)$. Despite the lack of sufficient post-surgical follow-up data regarding the effectiveness of the above surgeries, some reports mentioned that the surgeries enhanced motor development and significantly improved patient quality of life $(32,33)$. The patient in case 2 is an example of an individual who underwent a successful procedure.

Since cardiac abnormalities may be a direct cause of death in patients with RBS, cardiac surgery is necessary for patients diagnosed with severe congenital heart disease. However, some heart conditions may recur (3), and eligible patients and their families should be informed regarding the possible risks associated with surgery for congenital heart disease and the possible outcomes of such a surgery.

Sarcoma is a sporadically occurring tumor; however, it is more likely to occur in patients with congenital diseases than in patients without such diseases. These patients usually have poor prognoses. Thus, gaining local control of the disease rather than performing an extensive resection of the tumor may be advisable in such patients (34). The standard treatments for ophthalmological problems are also necessary, and special education intended to facilitate cognitive development should not be neglected.

Furthermore, patients with mild or negligible numbers of deformities are likely to lead normal lives and give birth to healthy children. These patients tend to be ignorant of their health conditions and may be misdiagnosed until more symptoms of the disease manifest $(3,35,36)$. It is necessary to determine how best to counsel these patients regarding the potential risks associated with adult manifestations of RBS, as well as the appropriate management of the disease. According to previous reports, adult cases of RBS often present with, spontaneous abortion, ocular findings beyond corneal clouding, and predispositions to cancer and thrombosis, as well as the typical symptoms of RBS $(16,35,36)$. When these features occur in youth, doctors should increase their index of suspicion for the diagnosis of mild RBS and should inform patients regarding other symptoms that they may experience. Problems such as heart failure, pulmonary failure, and tumorigenesis increase mortality rates in adult patients $(3,7)$. Consequently, thorough examinations of the cardiac, pulmonary, and renal systems; close monitoring for possible spontaneous abortions during pregnancy; continued ophthalmological follow-ups; and careful screenings for cancer and alterations in the parameters that determine the risk of veno-occlusive disease are necessary in adult patients with RBS/SC.

The primary objectives of all the treatments for RBS are to reconstruct and improve organ function, alleviate symptoms, and provide clinicians with more information with which they can guide future treatments. Fulfillment of this objective requires cooperation from multiple departments, including pediatrics, genetics, ophthalmology, cardiology, nephrology, neurology, child development, rehabilitation, general surgery, orthopedics and dentistry.

However, the best treatment for RBS is disease prevention. RBS can be easily identified on antenatal ultrasound examinations (37-39). Furthermore, cytogenetic analysis conducted using fetal DNA obtained from amniocytes after amniocentesis should be regarded as the gold standard test for 


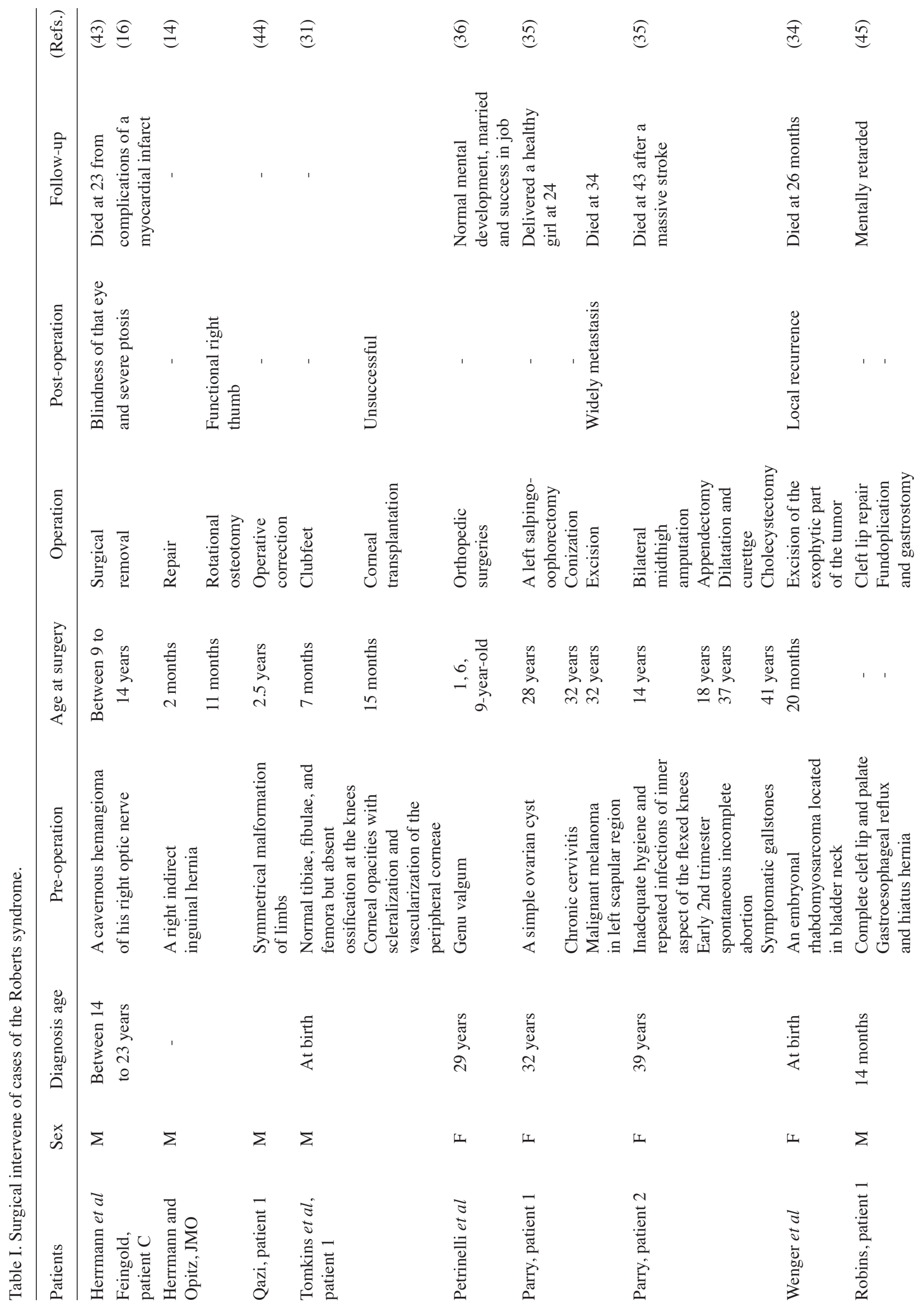




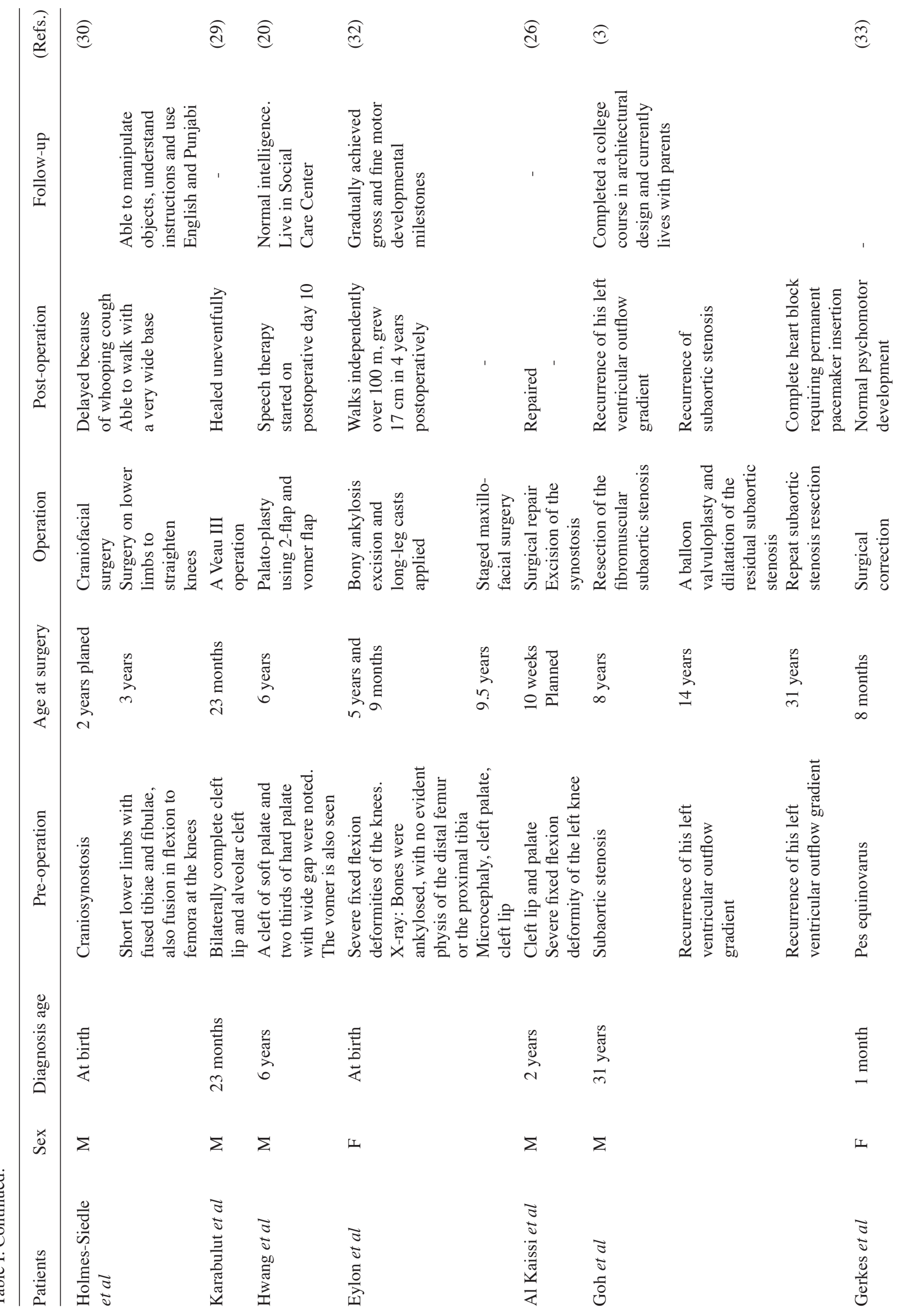


confirming the diagnosis of RBS during pregnancy (40-42). Therefore, regular prenatal examinations should be considered mandatory for patients hoping to avoid this rare but deadly congenital disease.

\section{Discussion}

Both patients displayed premature separation of the centromeres and craniofacial anomalies and limb deformities, findings that served as confirmation of the diagnosis of RBS. The male and female patients in this study had R:S ratios of 7:1 and 1:1, respectively. Consequently, the male patient presented with a phenotype of RBS, while the female patient present with a phenotype intermediate between RBS and SC. Regarding RS ratings, the male patient scored 1 and can thus can be considered to have severe disease. However, the female patient, scored 0 and exhibited multiple abnormalities, some of which were severe, and some of which were mild.

Under these circumstances, the RS rating is more reliable than the R:S ratio with respect to grading disease severity in patients with RBS. Since disease severity is strongly correlated with prognosis, we can safely assume that the female patient has a better prognosis than the male patient.

Both patients underwent surgeries during their infancy. Here, we evaluated the surgical outcomes for both patients. Although the male patient in this case underwent several craniofacial surgeries and a surgical repair of his cleft lip and palate between 1 and 2 years of age, he may require further treatment to address the following abnormalities: i) a limited cranial volume-restricting central nervous system development; ii) apparent exophthalmos, which normally requires a left frontal orbital transfer; and iii) an incompletely repaired cleft lip and adhesion of the upper lip to the maxilla, which restricts oral movement. However, the 7-year follow-up results of the craniofacial surgeries with which the patient was treated should indicate to surgeons that thorough examinations and proper preparation may enable some patients to withstand such craniofacial operations and experience improvements in their general conditions.

The female patient underwent cleft lip and palate repair between 1 and 2 years of age. She can communicate but has unclear speech, and the muscles of her tongue and lips require further training. At the age of 3, the patient received treatment for congenital clubfoot and experienced improvements in her motor abilities. Physicians witnessed improvements in the female patient's ability to perform self-care activities, as well as improvements in her social competence, over 11 years of follow-up. Both patients experienced significant benefits as a result of undergoing surgical correction, which not only improved their overall health conditions but also allowed them to fulfill their potential.

It should be noted is that when the female patient was born and presented with obvious deformities, her parents attributed her condition to fate. Thus, her doctor did not perform any additional assessments until the couple's fourth child presented with more severe disease. The findings of this study highlight the significance of ultrasound in pregnancy, as ultrasound can identify major anatomical abnormalities and birth defects. Furthermore, the male patient had an encephalocele and suffered from recurrent lung infections. If this patient had been born decades ago, he likely would not have survived through 
infancy. With the development of new medical techniques and increases in patient life expectancy, riskier interventions should be considered to enable patients to fulfill their potential.

In conclusion, RBS is a rare disease. Regular antenatal ultrasounds play a significant role in preventing this disease, especially in consanguineous couples. Without treatment, most patients with the disease die at young age. The results of the long-term follow-up of these two patients with severe deformities imply that proper surgical intervention at the appropriate age can improve quality of life and indicate that some patients may even lead normal lives. For patients with mild symptoms, close monitoring for the development of common adult-onset diseases can increase patient life expectancy and reduce mortality. It is our hope that this article will provide surgeons with evidence-based guidelines supporting the performance of surgeries that were once regarded as aggressive but may be beneficial in patients with RBS.

\section{References}

1. Roberts J: A child with double cleft of lip and palate, protrusion of the intermixillary portion of the upper jaw and imperfect development of the bones of the four extremitis. Ann Surg 70: 252-254, 1919

2. Gordillo M, Vega $\mathrm{H}$ and Jabs EW: Roberts syndrome. In: GeneReviews. Pagon RA, Adam MP, Ardinger HH, Wallace SE, Amemiya A, Bean LJH, Bird TD, Ledbetter N, Mefford HC, Smith RJH and Stephens K (eds). University of Washington, Seattle, WA, 1993.

3. Goh ES, Li C, Horsburgh S, Kasai Y, Kolomietz E and Morel CF: The Roberts syndrome/SC phocomelia spectrum-a case report of an adult with review of the literature. Am J Med Genet A 152A $472-478,2010$.

4. Ivanov D, Schleiffer A, Eisenhaber F, Mechtler K, Haering CH and Nasmyth K: Ecol is a novel acetyltransferase that can acetylate proteins involved in cohesion. Curr Biol 12: 323-328, 2002.

5. Tanaka K and Watanabe Y: Chromatid cohesion: Acetylation joins the sisters. Curr Biol 18: R917-R919, 2008.

6. Unal E, Heidinger-Pauli JM and Koshland D: DNA double-strand breaks trigger genome-wide sister-chromatid cohesion through Ecol (Ctf7). Science 317: 245-248, 2007.

7. Hou F and Zou H: Two human orthologues of Ecol/Ctf7 acetyltransferases are both required for proper sister-chromatid cohesion. Mol Biol Cell 16: 3908-3918, 2005.

8. Gordillo M, Vega H, Trainer AH, Hou F, Sakai N, Luque R, Kayserili H, Basaran S, Skovby F, Hennekam RC, et al: The molecular mechanism underlying Roberts syndrome involves loss of ESCO2 acetyltransferase activity. Hum Mol Genet 17: 2172-1280, 2008.

9. O'Brien HR and Mustard HS: An adult living case of total phocomelia. J Am Med Assoc 77: 1964-1967, 1921.

10. Freeman MV, Williams DW, Schimke RN, Temtamy SA, Vachier E and German J: The Roberts syndrome. Clin Genet 5: 1-16, 1974.

11. Leonard P, Rendle-Short J and Skardoon L: Roberts'-SC phocomelia syndrome with cytogenetic findings. Hum Genet 60 379-380, 1982.

12. Stanley WS, Pai GS, Horger EO III, Yan YS and McNeal KS: Incidental detection of premature centromere separation in amniocytrs associated with a mild form of Roberts syndrome. Prenat Diagn 8: 565-569, 1988.

13. Judge C: A sibship with the pseudothalidomide syndrome and an association with $\mathrm{Rh}$ incompatibility. Med J Aust 2: 280-281, 1973.

14. Herrmann J and Opitz JM: The SC phocomelia and the Roberts syndrome: Nosologic aspects. Eur J Pediatr 125: 117-134, 1977.

15. Van Den Berg DJ and Francke U: Roberts syndrome: A review of 100 cases and a new rating system for severity. Am J Med Genet 47: 1104-1023, 1993.

16. Feingold M: History of C-patient with SC-Roberts/pseudothalidamide syndrome. Am J Med Genet 43: 898-899, 1992.

17. Waldenmaier C, Aldenhoff $\mathrm{P}$ and Klemm T: The Roberts syndrome. Hum Genet 40: 345-349, 1978.
18. Hall BD and Greenberg MH: Hypomelia-hypotrichosis-facial hemangioma syndrome (pseudothalidomide, SC syndrome, SC phocomelia syndrome). Am J Dis Child 123: 602-604, 1972.

19. Jabs EW, Tuck-Muller CM, Cusano R and Rattner JB: Studies of mitotic and centromeric abnormalities in Roberts syndrome: Implications for a defect in the mitotic mechanism. Chromosoma 100: 251-261, 1991.

20. Hwang K, Lee DK, Lee SI and Lee HS: Roberts syndrome, normal cell division, and normal intelligence. J Craniofac Surg 13: 390-394, 2002.

21. Vega H, Waisfisz Q, Gordillo M, Sakai N, Yanagihara I, Yamada M, van Gosliga D, Kayserili H, Xu C, Ozono K, et al: Roberts syndrome is caused by mutations in ESCO2, a human homolog of yeast ECO1 that is essential for the establishment of sister chromatid cohesion. Nat Genet 37: 468-470, 2005.

22. Percival SM, Thomas HR, Amsterdam A, Carroll AJ, Lees JA, Yost HJ and Parant JM: Variations in dysfunction of sister chromatid cohesion in esco2 mutant zebrafish reflect the phenotypic diversity of Roberts syndrome. Dis Model Mech 8: 941-955, 2015.

23. Schüle B, Oviedo A, Johnston K, Pai S and Francke U: Inactivating mutations in ESCO2 cause SC phocomelia and Roberts syndrome: No phenotype-genotype correlation. Am J Hum Genet 77: 1117-1128, 2005.

24. Vega H, Trainer AH, Gordillo M, Crosier M, Kayserili H, Skovby F, Uzielli ML, Schnur RE, Manouvrier S, Blair E, et al: Phenotypic variability in 49 cases of ESCO 2 mutations, including novel missense and codon deletion in the acetyltransferase domain, correlates with ESCO2 expression and establishes the clinical criteria for Roberts syndrome. J Med Genet 47: 30-37, 2010.

25. Holden KR, Jabs EW and Sponseller PD: Roberts/pseudothalidomide syndrome and normal intelligence: Approaches to diagnosis and management. Dev Med Child Neurol 34: 534-539, 1992.

26. Al Kaissi A, Csepan R, Klaushofer K and Grill F: Femoraltibial-synostosis in a child with Roberts syndrome (Pseudothalidomide): A case report. Cases J 1: 109, 2008.

27. Mossey PA, Little J, Munger RG, Dixon MJ and Shaw WC: Cleft lip and palate. Lancet 374: 1773-1785, 2009.

28. Temtamy SA and Loutfi A: Some genetic and surgical aspect of the cleft lip-cleft palate in Egypt. Cleft Palate J 7: 578-594, 1970.

29. Karabulut AB, Aydin H, Erer M, Mezdegi A and Güven E: Roberts syndrome from the plastic surgeon's viewpoint. Plast Reconstr Surg 108: 1443-1445, 2001.

30. Holmes-Siedle M, Seres-Santamaria A, Crocker M, Hall JG and Crouchman M: A sibship with Roberts/SC phocomelia syndrome. Am J Med Genet 37: 18-22, 1990.

31. Tomkins D, Hunter A and Roberts M: Cytogenetic findings in Roberts-SC phocomelia syndrome (s). Am J Med Genet 4: 17-26, 1979.

32. Eylon S, Beeri M, Joseph K and Meyer S: Femorotibial ankylosis in a child with Roberts syndrome: An 'aggressive' approach to habilitation. J Pediatr Orthop 27: 926-929, 2007.

33. Gerkes EH, van der Kevie-Kersemaekers AM, Yakin M, Smeets DF and van Ravenswaaij-Arts CM: The importance of chromosome studies in Roberts syndrome/SC phocomelia and other cohesinopathies. Eur J Med Genet 53: 40-44, 2010.

34. Wenger SL, Blatt J, Steele MW, Lloyd DA, Bellinger M, Phebus CK, Horn M and Jaffe R: Rhabdomyosarcoma in Roberts syndrome. Cancer Genet Cytogenet 31: 285-289, 1988.

35. Parry DM, Mulvihill JJ, Tsai SE, Kaiser-Kupfer MI and Cowan JM: SC phocomelia syndrome, premature centromere separation, and congenital cranial nerve paralysis in two sisters, one with malignant melanoma. Am J Med Genet 24: 653-672, 1986.

36. Petrinelli P, Antonelli A, Marcucci L and Dallapiccola B: Premature centromere splitting in a presumptive mild form of Roberts syndrome. Hum Genet 66: 96-99, 1984.

37. Socolov RV, Andreescu NI, Haliciu AM, Gorduza EV, Dumitrache F, Balan RA, Puiu M, Dobrescu MA and Socolov DG: Intrapartum diagnostic of Roberts syndrome - case presentation. Rom J Morphol Embryol 56: 585-588, 2015.

38. Dulnuan DJ, Matsuoka M, Uketa E, Hayashi K, Murotsuki J, Nishimura G and Hata T: Antenatal three-dimensional sonographic features of Roberts syndrome. Arch Gynecol Obstet 284: 241-244, 2011.

39. Paladini D, Palmieri S, Lecora M, Perone L, Di Meglio A, D'Armiento M, Cascioli C and Martinelli P: Prenatal ultrasound diagnosis of Roberts syndrome in a family with negative history. Ultrasound Obstet Gynecol 7: 208-210, 1996. 
40. Schulz S, Gerloff C, Ledig S, Langer D, Volleth M, Shirneshan K and Wieacker P: Prenatal diagnosis of Roberts syndrome and detection of an ESCO2 frameshift mutation in a Pakistani family. Prenat Diagn 28: 42-45, 2008.

41. Benzacken B, Savary JB, Manouvrier S, Bucourt M and Gonzales J: Prenatal diagnosis of Roberts syndrome: Two new cases. Prenat Diagn 16: 125-130, 1996.

42. Stioui S, Privitera O, Brambati B, Zuliani G, Lalatta F and Simoni G: First-trimester prenatal diagnosis of Roberts syndrome. Prenat Diagn 12: 145-149, 1992.

43. Herrmann J FM, Tuffli G and Opitz J: A familial dysmorphogenetic syndrome of limb deformities, characteristic facial appearance and associated anomalies: the "pseudothalidomide" or "SC-syndrome.”. Birth Defects Orig Artic Ser 5: 81-89, 1969.
44. Qazi QH, Kassner EG, Masakawa A, Madahar C and Choi SJ: The SC phocomelia syndrome: report of two cases with cytogenetic abnormality. Am J Med Genet 4: 231-238, 1979.

45. Robins DB, Ladda RL, Thieme GA, Boal DK, Emanuel BS, Zackai EH: Prenatal detection of Roberts-SC phocomelia syndrome: report of 2 sibs with characteristic manifestations. Am J Med Genet 32: 390-394, 1989

46. Ragavan M, Reddy S, Kumar C: Tetra-amelia with lung hypoplasia and facial clefts, Roberts-SC syndrome: report of two cases. Pediatr Surg Int 26: 1049-1052, 2010. 